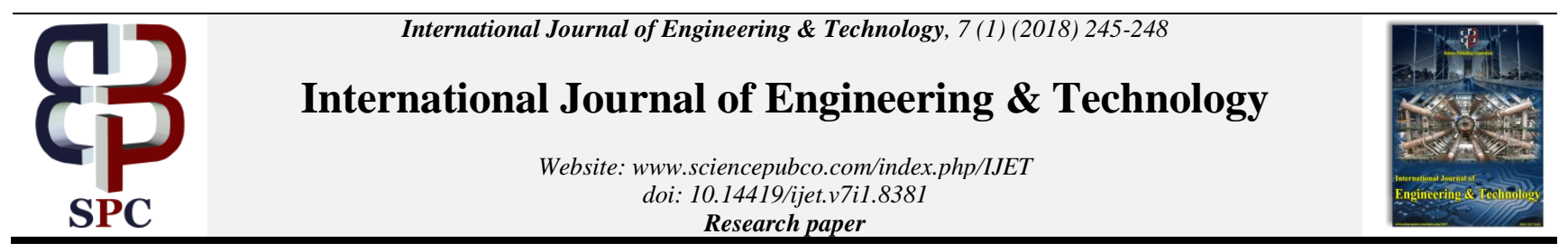

\title{
Efficient OFDM transceiver design for VLC systems in fading channel
}

\author{
Jayakumari. J ${ }^{1 *}$, Rakhi K. J ${ }^{2}$ \\ ${ }^{1}$ Department of ECE, Mar Baselios College of Engineering and Technology, Thiruvananthapuram, Kerala \\ ${ }^{2}$ Department of ECE, Noorul Islam Centre for higher Education, Kumaracoil, Tamil Nadu \\ *Corresponding author E-mail: jayakumarij72@gmail.com
}

\begin{abstract}
With the widespread effective usage of LEDs the visible light communication (VLC) system has brought out an increasing interest in the field of wireless communication recently. VLC is envisioned to be an appealing substitute to RF systems because of the advantages of LEDs such as high communication security, rich spectrum, etc. For achieving bearable inter symbol interference (ISI) and high data rates, OFDM can be employed in VLC. In this paper, the performance of VLC system with popular unipolar versions of OFDM viz. Flip-OFDM and ACO-OFDM is analyzed in fading channels. From the simulation results it is seen that the Flip-OFDM-VLC system outperforms the ACO-OFDM-VLC system in terms of bit error rate and is well suited for future $5 \mathrm{G}$ applications.
\end{abstract}

Keywords: 5G; ACO-OFDM; Flip-OFDM; LTE; VLC.

\section{Introduction}

The demand for wireless transmission systems with fast connections has skyrocketed in the recent years. Radio frequency (RF) communication is used mostly for wireless communication, especially for indoor communications [10]. The limitations of the RF communication systems such as limited bandwidth, regulated frequency spectrum, uncontrolled radiation, etc. [4] are leading to the necessity of other communication methods. As an effective solution to achieve wireless connectivity with high data rate, several techniques have been explored. Visible Light technology is an attractive form of communication for high-speed indoor links [1]-[3].

Visible Light communication (VLC) is envisioned to be an appealing substitute to RF systems because of the effective usage of light emitting diodes [1], [9]. VLC can support illumination and communication simultaneously and offers the benefits of high rates, low cost, safety, security, and no electronic interference. Therefore, VLC has been regarded as an important supplement to, or replacement of, traditional RF communication in indoor environments [5][8].To fulfill high-speed transmissions, orthogonal frequency-division multiplexing (OFDM), which has been widely used in 4G systems (e.g., LTE), has also been considered in VLC . However OFDM symbols are bipolar. Since the intensity modulation with direct detection (IM/DD) is used in VLC systems, the transmitting signals must be real and nonnegative [1] - [2].Hence the OFDM symbols should be converted to unipolar format before applying to a VLC system. Several techniques are available to convert bipolar OFDM to unipolar. They are DC biased optical OFDM (DCOOFDM), PAM-modulated discrete multitone (PAM-DMT), Asymmetrically Clipped optical OFDM (ACO-OFDM) [1] - [3], [5]. Most popular among them are ACO-OFDM and DCO-OFDM. Recently, a novel technique called Flip-OFDM is proposed [1]-[3]. In Flip-OFDM [4], the real bipolar signal is decomposed into positive and negative parts [1]- [3], [9], [10] which are extracted from real bipolar signal by preserving the Hermitian symmetry property
[1]- [3], [10]. The polarity of the negative part is flipped and both the parts are transmitted over two consecutive OFDM sub frames [1]-[3]. The Digital Fourier Transform properties and the frame properties of OFDM are exploited for obtaining the unipolar signal without biasing [3], [8], [9].

\section{Proposed VLC system}

This paper deals with the design of an efficient OFDM scheme for VLC system. Two unipolar versions of OFDM viz. ACO-OFDM and Flip-OFDM are attempted. Both techniques can be used to compensate multipath distortion effects in optical wireless channels.

\subsection{ACO-OFDM-VLC transceiver}

Asymmetric clipped optical OFDM can reduce the optical power requirement for a given data rate because no optical power is wasted in DC-biasing a bipolar OFDM waveform to become unipolarnegative values are simply set to zero. ACO-OFDM utilizes odd subcarriers for modulation. At the transmitter (Fig. 1) the 16-bit random symbols (i.e., random numbers between 0 and 15) is given as input. These random symbols are then modulated using 16 QAM. Let $M_{k}$ be the transmitted QAM symbol in the $k^{\text {th }}$ subcarrier of the OFDM. The QAM symbols are mapped onto the first half of OFDM odd subcarriers, where $k=0,1,2, \ldots, \frac{k}{4}-1$ and even subcarriers are set to zero, i.e.

$$
M_{2 k}=0, \quad k=0,1,2, \ldots, \frac{k}{2}
$$




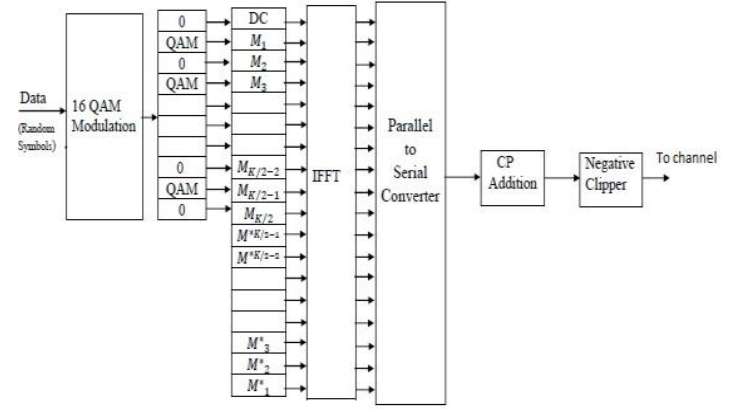

Fig. 1: ACO-OFDM-VLC Transmitter

Thus the DC component and the $\frac{k^{\text {th }}}{2}$ OFDM subcarrier becomes zero. Hermitian symmetry property is applied to the transmitted QAM symbols, i. e,

$M_{k}=M_{K-k}^{*}, \quad k=0,1,2, \ldots, \frac{k}{2}-1$

Where $*$ denotes the complex conjugation. At the $g^{\text {gh }}$ time instant, the output of the IFFT operation is given by

$m(g)=M_{0}+\sum_{K=1}^{\frac{K}{2}-1} M_{k} \exp \left(\frac{j \pi k g}{K}\right)+M_{\frac{k}{2}} \exp (j 2 \pi g)+\sum_{\frac{K}{2}+1}^{K-1} M_{K-k}^{*} \exp \left(\frac{j \pi k g}{K}\right)$

Where $K, M_{0}, M_{k}, M_{k}^{*}$, represent the IFFT size, DC component, QAM symbol of center carrier, the conjugate symmetry of the QAM symbol $M_{k}$ respectively. $M_{\frac{k}{2}}$ and are set to zero in order to avoid any residual complex component or DC shift. The signal obtained by the IFFT operation has odd symmetry property

$$
m[l]=-m\left[g+\frac{k}{2}\right]
$$

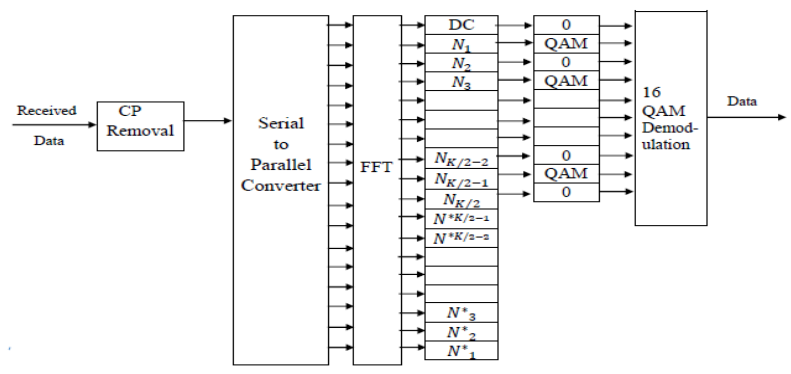

Fig. 2: ACO-OFDM-VLC Receiver.

Because of the above odd symmetry property, the clipping of the negative samples of $m[l]$ without the destruction of the original information is possible after the parallel to serial conversion and the cyclic prefix (CP) addition. The clipping of the negative samples of the signal is done using a negative clipper. The obtained clipped signal $m_{c}(h)$ is defined as

$m_{c}(h)=\begin{array}{ll}m(h), & \text { if } m(h)^{3} \\ 0 & 0\end{array}$

This signal is then transmitted through Rayleigh fading channel. At the receiver (Fig. 2), after removing the cyclic prefix, FFT of the signal is found. The output of the odd subcarriers of the first half of the FFT carriers are then given to QAM demodulator and the original data is obtained. In ACO-OFDM, since optical power is not wasted and power requirement is less.

\subsection{Flip-OFDM-VLC transceiver}

The concept of Flip-OFDM is quite similar to ACO-OFDM except that in Flip-OFDM the polarity of negative part is inverted after IFFT operation (Fig.3). Flip-OFDM/U-OFDM has double the initial latency to decode a single information frame because two consecutive frames need to be received in order for the demodulation process to begin. The FFT size of U-OFDM/Flip-OFDM is half the FFT size of ACO-OFDM.

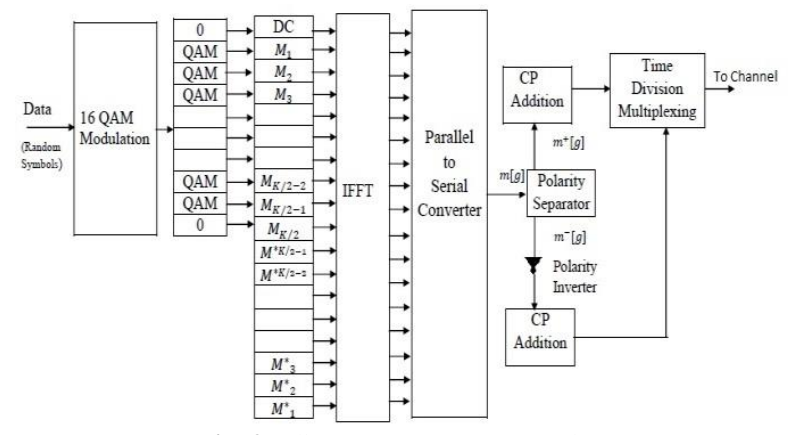

Fig. 3: Flip-OFDM-VLC Transmitter.

Let $m[g]$ repesent the time domain signal at the output of IFFT block and will be bipolar in nature and can be represented as

$$
m[g]=m^{+}[g]+m^{-}[g]
$$

where the positive part is defined as

$m^{+}[g]= \begin{cases}m[g], & \text { if } m[g] \geq 0 \\ 0, & \text { otherwise }\end{cases}$

And the negative part is defined as

$m^{-}[g]=\left\{\begin{array}{l}m[g], \text { if } m[g]<0 \\ 0, \quad \text { otherwise }\end{array}\right.$

The decomposition of real time domain signal is done by using a polarity separator as shown in Fig. 4. The negative part $m^{-}[g]$ is then flipped (polarity inverted) to obtain $-m^{-}[g]$ using an inverter. Both the parts are transmitted separately over two consecutive OFDM frames by using time division multiplexing (TDM). The positive part is transmitted in the positive OFDM subframe and the polarity inverted negative part is transmitted in the negative OFDM subframe.The CP of length $\alpha$ samples is added to each OFDM subframe. Thus the second OFDM subframe is delayed by $(k+\alpha)$ samples and hence transmitted after the positive OFDM subframe. At the reception side (Fig. 4), the received data is de-multiplexed and the $\mathrm{CP}$ is removed from each OFDM sub frame and the polarity of the second sub frame is inverted by the polarity inverter. The original bipolar real time signal is obtained as

$n[g]=n_{1}[g]+n_{2}[g]$

The obtained bipolar signal is then converted to parallel form and is fed to the FFT block. The output of first half of the FFT carriers is the QAM modulated signal and that of the second half is the complex conjugate of the QAM modulated signal. The QAM modulated signals are then demodulated to get the original data. 


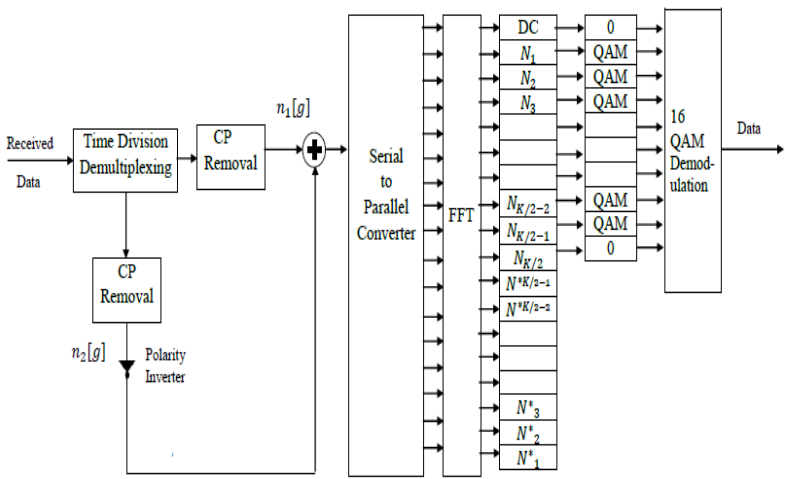

Fig. 4: Flip-OFDM-VLC Receiver.

\section{Simulation, results and discussion}

The simulation of the proposed ACO-OFDM-VLC transceiver and Flip-OFDM-VLC transceiver was done using Matlab software. The 16-bit random digital message was given as input to both. The channel models used for simulation are Rayleigh with 5 taps and Doppler shift $150 \mathrm{~Hz}$ and Rayleigh with 2 taps and Doppler shift $80 \mathrm{~Hz}$. In ACO-OFDM, after the 16-QAM modulation of the data, zero padding was done to make the data of length 32-bit. Here zero padding was done in an alternate manner, i.e. to the data applied to odd numbered subcarriers zero padding was performed. Then the conjugate of the zero padded QAM modulated data was then taken to preserve Hermitian symmetry property. Then this signal was fed as input to the first half (first 64) of the IFFT subcarriers and the conjugated zero padded QAM modulated data to the second half (last 64) of the IFFT subcarriers. After converting to time domain, the signal was converted from parallel to serial form. The 16-bit cyclic prefix $(\mathrm{CP})$ was then added at the end of the serially converted data and the negative samples of the data are clipped by using a negative clipper and then transmitted.

In Flip-OFDM, after the QAM modulation, zero padding was performed at the end of the data for all subcarriers. Then the conjugate of the zero padded QAM modulated data was then taken to preserve Hermitian symmetry property. Then this signal was fed as input to the first half (first 64) of the IFFT subcarriers and the conjugated zero padded QAM modulated data to the second half (last 64) of the IFFT subcarriers. The output of the IFFT block is converted to serial form and was decomposed into positive and negative parts using the polarity separator and the polarity of the negative parts are flipped. The 8-bit CP is then added to both positive and flipped negative parts and are time division multiplexed and are transmitted. The bit error rate (BER) performance of the proposed schemes is plotted in Fig. 7. It is seen that the BER of Flip-OFDM is lower compared to ACO-OFDM system in fading channels.

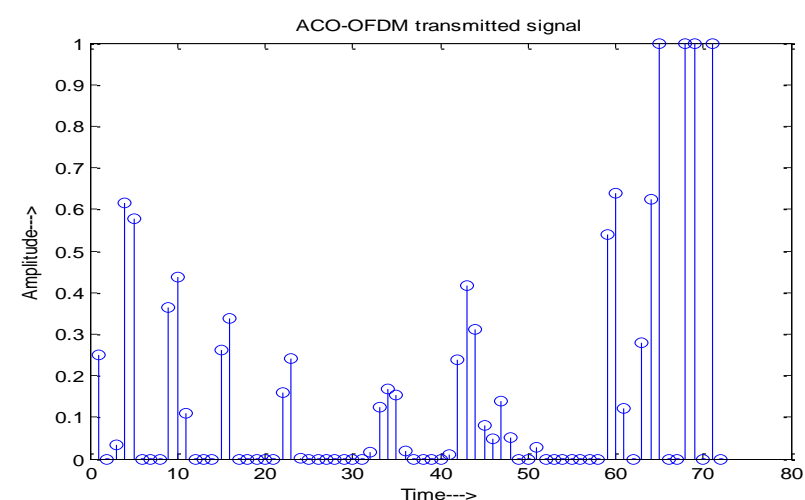

Fig. 5: ACO-OFDM Transmitted Signal.

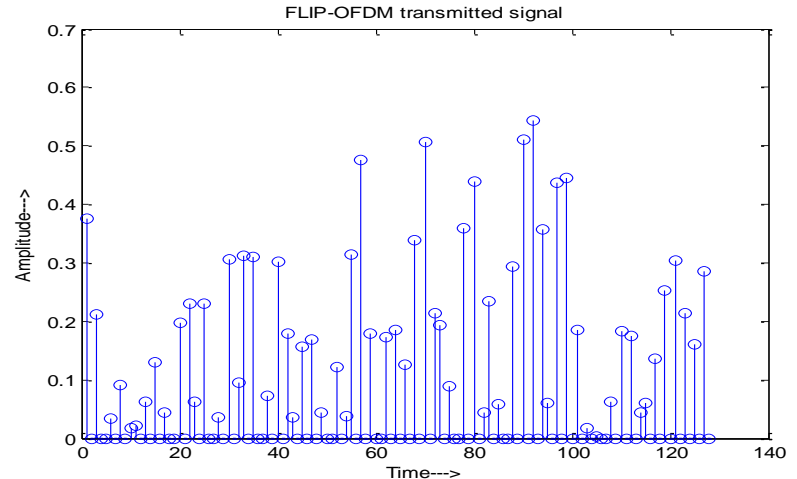

FIG. 6: FLIP-OFDM Transmitted Signal.

\section{Conclusion}

This paper focuses on designing an efficient transmission scheme for VLC using OFDM. The performance of VLC system with popular unipolar versions of OFDM viz. Flip-OFDM and ACO-OFDM is analyzed. From the simulation results it is seen that the FlipOFDM-VLC system outperforms the ACO-OFDM-VLC system in terms of BER. At a SNR of 20dB, the BER of Flip-OFDM OWC system is 0.0928 and that of ACO-OFDM OWC system is 0.1835 . Also, it is seen that the VLC system employing OFDM can provide high data rate. Even though the hardware complexity of FlipOFDM-VLC system is slightly higher compared with the ACOOFDM-VLC system, Flip-OFDM scheme is the best transmission scheme for the VLC system because of its high data rate and reduced bit error rate performance.

(A)

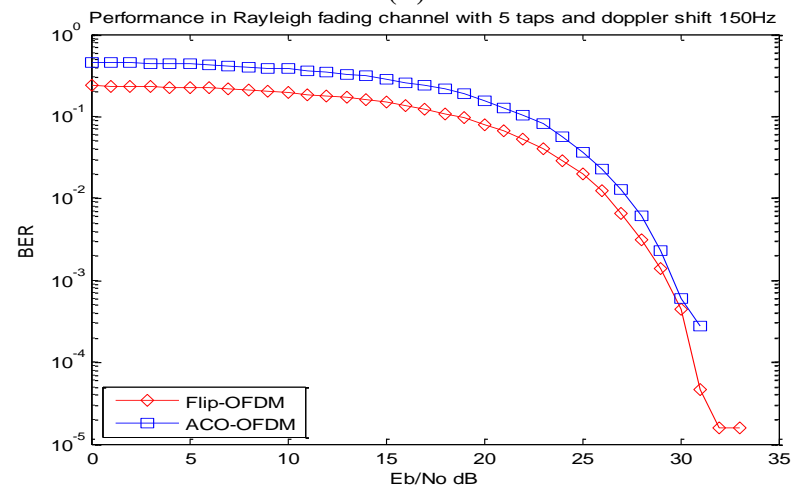

(B)

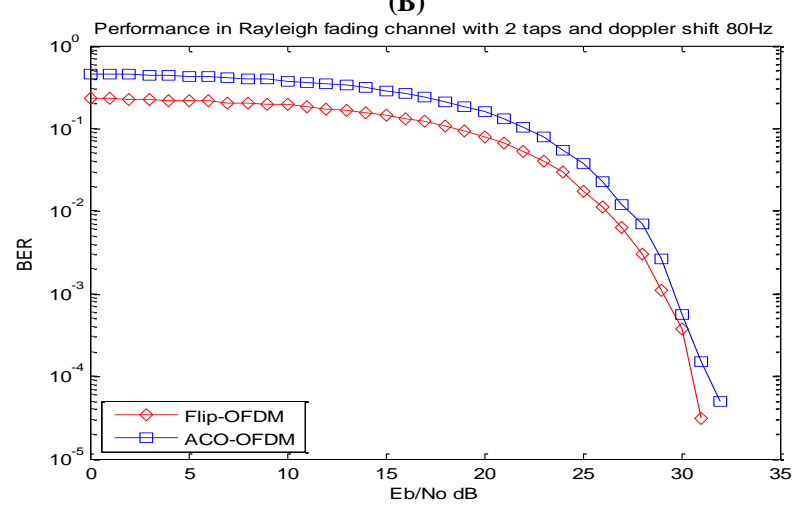

Fig. 7: Bit error rate comparison of ACO-OFDM and flip-OFDM VLC system. 


\section{References}

[1] Nuo Huang, Jun-Bo Wang, Cunhua Pan, Jin-Yuan Wang, Yijin Pan, and Ming Chen, "Iterative Receiver for Flip-OFDM in Optical Wireless Communication", in IEEE Photonics Technology Letters, Vol. 27, No. 16, pp. 17-29, August 15, 2015. https://doi.org/10.1109/LPT.2015.2438338.

[2] N. Fernando, Y. Hong, and E. Viterbo, "Flip-OFDM for optical wireless communications," in Proc. IEEE Inf. Theory Workshop, pp. 5 9, Oct. 2011. https://doi.org/10.1109/ITW.2011.6089566.

[3] D. Tsonev and H. Haas, "Avoiding spectral efficiency loss in unipolar OFDM for optical wireless communication," in Proc. IEEE Int Conf. Commun. (ICC), pp. 3336-3341, Jun. 2014 https://doi.org/10.1109/ICC.2014.6883836.

[4] D. J. F. Barros, S. K. Wilson, and J. M. Kahn, "Comparison of orthogonal frequency-division multiplexing and pulse-amplitude modulation in indoor optical wireless links," IEEE Trans. Commun., vol. 60, no. 1, pp. 153-163, Jan. 2012 https://doi.org/10.1109/TCOMM.2011.112311.100538.

[5] Poompat Saengudomlert, "On the Benefits of Pre-Equalization for ACO-OFDM and Flip-OFDM Indoor Wireless Optical Transmissions Over Dispersive Channels, ’Journal of Lightwave Technology, vol. 32, no. 1, Jan. $1, \quad 2014$. https://doi.org/10.1109/JLT.2013.2286746.

[6] Jaya kumari.J and Sakuntala.S.Pillai, "Performance of multicarrier OFDM systems" Proc. 36th IETE Mid Term Symposium on Emerging and Futuristic Communication Systems (EFCoS-05), pp.347-352, 30 Apr-01 May 2005.

[7] Jaya kumari.J and Sakuntala.S.Pillai, "Orthogonal Frequency Division Multiplexing" Proc. 17th Kerala Science Congress, KFRI, Peechi, pp.139-142, 29-31 Jan 2005

[8] Mohammad Ali Khalighi, and Murat Uysal," Survey on Free Space Optical Communication: A Communication Theory Perspective," IEEE Communication Surveys \& Tutorials, vol. 16, no. 4, Fourth Quarter,June. 2014.

[9] Murat Uysal, and Hatef Nouri," Optical wireless communications an emerging technology," IEEE 16th International Conference on Transparent Optical Networks, July 2014

[10] Zhenhua Yu, Robert J. Baxley, and G. Tong Zhou,” Achievable data rate analysis of clipped FLIP-OFDM in optical wireless communication," IEEE Globecom Workshops, pp. 1203-1207, Dec. 2012. 